\title{
A Novel Functional Impression Procedure for Distal-Extension Removable Partial Dentures
}

\author{
$\mathrm{Li} \mathrm{QL}^{*}, \mathrm{Cao} \mathrm{Y}$ \\ Department of Prosthodontics, College of Stomotology, Anhui Medical University, Hefei, China.
}

Case Report

\section{Abstract}

Background: Functional selective pressure impression on the residual ridge is much highly recommended than the rest of the anatomic impressions in distal-extension removable partial dentures (RPD). However, the present methods are significantly more complex, require additional treatment time, and involve more laboratory procedures.

Methods: This novel impression procedure involves the following steps. A special force transport unit is first designed and assembled on the stock or custom tray over the residual ridge, through which the opposite teeth can bite on the force transport unit and transport the occlusion force to the impression body. The primary full arch impression is then created using heavy body type of vinyl polysiloxane impression materials. Next, part of the impression overlapping the residual ridge is freed from the intact impression body and the tray with a scalpel. Finally, the final impression is made with a lightbody vinyl polysiloxane impression. The light-body materials are loaded onto the surface of the primary impression and the space from the scalpel cut. The primary impression is set into the mouth, allowing the opposite teeth to bite on the force transport during the impression making process. The free parts are bound to obtain the final impression after the materials are set.

Results: This functional impression method could record the anatomic form of the teeth and the functional form of the residual ridge, simultaneously capturing the surface morphology and location of the saddle, which is derived from the deformation after chew loading.

Conclusion: This functional pressure impression for RPD is simple, could be easily mastered by dentists, and does not increase the laboratory workload of dental technicians.

\author{
*Corresponding Author: \\ Dr. Quan-Li Li, \\ Tel/Fax: 86-0551-5118677 \\ E-mail: ql-li@126.com \\ Received: November 09, 2015 \\ Accepted: November 21, 2015 \\ Published: November 23, 2015
}

Department of Prosthodontics, College of Stomotology, Anhui Medical University, No.69 Meishan Road Hefei, China.CA 230032.

Citation: Li QL, Cao Y (2015) A Novel Functional Pressure Impression Procedure for Distal-Extension Removable Partial Dentures. Int J Dentistry Oral Sci. 2(11), 168-172. doi: http://dx.doi.org/10.19070/23778075-1500035

Copyright: Li QL ${ }^{\odot}$ 2015. This is an open-access article distributed under the terms of the Creative Commons Attribution License, which permits unrestricted use, distribution and reproduction in any medium, provided the original author and source are credited.

\section{Introduction}

Functional selective pressure impression overlapping the residual ridge is much highly recommended than the rest of the mucostatic anatomic impression in distal-extension removable partial dentures (RPD)[1-5]. Functional impression for RPD requires recording of the anatomic form of the teeth and the functional form of the residual ridge under occlusal force. The key is to capture the surface morphology and location of the saddle, which is derived from deformation after loading. The technique was previously characterised in the following manner $[6,7]$. The sectional functional impression of the residual ridge is obtained using a section fragment custom tray with impression materials (such as wax or thermoplastic modelling compound) that are held under pressure with mastication forces. The form is then taken out, trimmed, and placed into the mouth again overlying the residual ridges in situ. Afterward, final impression materials are loaded into the stock tray or custom tray to obtain the whole dental arch impression containing the remaining tooth and the residual ridge. This method can only reflect the surface morphology of the ridge under its functional state; however, the location of the ridge surface under its functional state is compromised because the resilient mucosa will recover after the mastication forces are unloaded during the secondary impression. The altered cast technique has been recently more highly recommended to obtain the functional impression. This technique is described through the following steps [8]. The metal framework is made according to a full arch anatomic impression. The secondary functional impression of the residual ridge is made with a self-curing resin tray attached to the framework, overlying the ridge under the mastication force. The denture-based area of the master cast is cut off and discarded. The framework with the secondary impression is placed on the master cast, and the artificial stone is poured in the edentulous area. The altered cast impressions can simultaneously capture the surface contour and location of the ridge under mastication function and most effectively fulfil the requirements of relating the anatomic form of the teeth to the functional form of the residual ridge. However, this method requires additional treatment time and the laboratory procedures involved are difficult. Errors may be introduced when orienting and stabilising the framework on the sectioned cast. Thus, an altered cast trial has 
been applied to simplify the procedure $[4,5,9,11]$. Dumbrigue $\mathrm{HB}$ [12] reported a simple selective-pressure single impression procedure for tooth-mucosa-supported RPD. However, the pressure force in this method comes from the fingers rather than from the mastication force. Thus, this method is not considered a truly functional impression. The current study presents a novel simple functional impression procedure that relates the anatomic form of the teeth to the functional form of the residual ridges in distal extension.

\section{Materials and Methods}

\section{Case 1}

A 53-year-old man with a chief complaint of missing the molars of the maxillary region was referred to our hospital in September 2014. Deciduous teeth had been previously extracted because of periodontal disease. The patient had no history of trauma and systemic disease. The patient needed a removable partial denture for functional and aesthetic reasons. The following impression procedure was carried out (Figure 1-6).

\section{Case 2}

A 61-year-old man was referred to our hospital for restoration of the bilateral low molars in March 2015. An attachment denture protocol was carried out after the fixed part of the denture was fabricated and tried in (Figure 7). The following impression procedure was performed to create functional impression for the patient and fabricate the removable part of the denture (Figures 7-11).

Fabrication of the assembly unit for masticatory force transport: A metal wire with a diameter of approximately 1-2 $\mathrm{mm}$ or resin with other hardening materials can be used to fabricate the assembly unit (Figure 1). The force transport unit contains two parts: the retention form, which is buried into the impression ma- terial overlying the ridge and a narrow bar (cylinder), which passes through a perforated stock or custom tray and the opposite tooth can bite on.

Creation of a preliminary impression: The impression with a stock tray is used as an example.

A proper stock tray is selected. The stock tray is made of stainless steel with an even perforation and an approximately $2 \mathrm{~mm}$ diameter distribution. The force transport unit is assembled onto the tray (Figures 2 and 8), and its location is over the residual ridge. The heavy body type of the vinyl polysiloxane (VPS) impression materials is used for the primary impression. Before the impression material is set, the direction of the bar of the force transport unit is adjusted to allow the opposite tooth to bite on it and thereby finish the border moulding operation (Figures 3 and 9).

The part of the impression that overlaps the residual ridge is freed from the intact impression body: The impression body is completely cut along the boundary of the residual ridge with a scalpel or other knife to free the impression overlapping the residual ridge from the impression body and the tray (Figures 4 and 10) while retaining the body in situ. If a force is applied onto the bar of the force transport unit, the section impression overlapping the residual ridge can freely move in a vertical direction.

Make the final impression: The light-body PVS impression material is placed onto the tissue surface of the primary impression. The impression material is placed into the space from the scalpel cut (Step 3) and the perforation of the tray to keep the free portion of the primary impression body (Step 3) bonded to the tray. The tray is then fitted into the mouth, and the opposite teeth bite is retained on the bar of the force transport until the material sets (Figures 5 and 11) and the border moulding operation is completed. The final impression that reflects the anatomic form of the teeth to the functional form of the residual ridges is obtained in distal extension (Figures 6 and 12).

Figure 1. Design of the assembly unit for masticatory force transport.

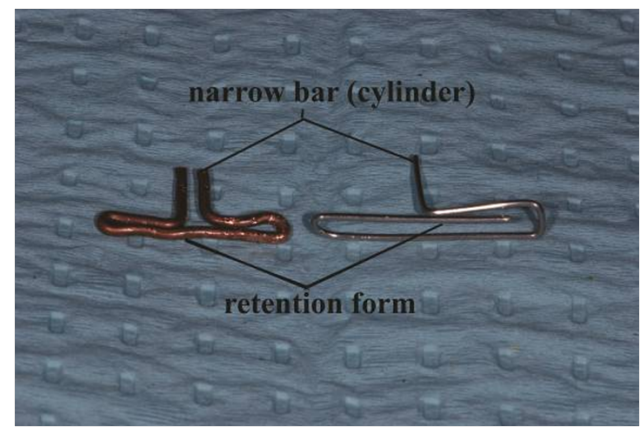

Figure 2. The force transport unit was assembled on the perforated stock tray (Case 1).

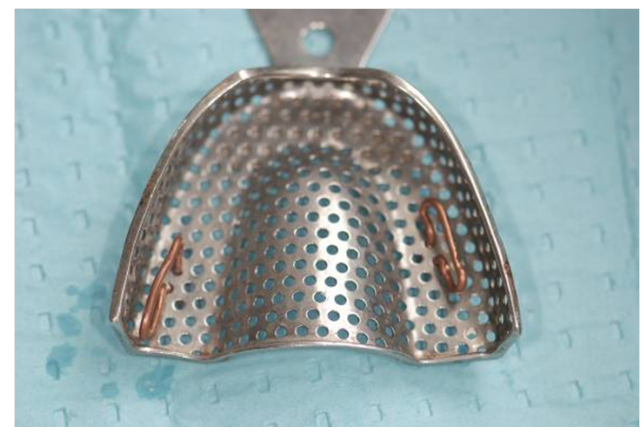


Figure 3. The preliminary impression was made from heavy body-type of vinyl polysiloxane impression materials; (a) tissue surface view; (b) outside view showing the relationship between the bar of the force transport unit and the impression body (Case 1).

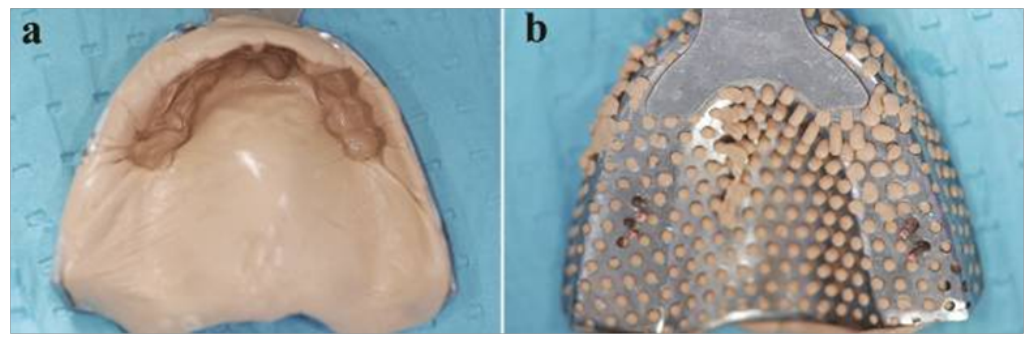

Figure 4. Part of the impression overlapping the residual ridge freed from the whole impression body with a scalpel (Case 1).

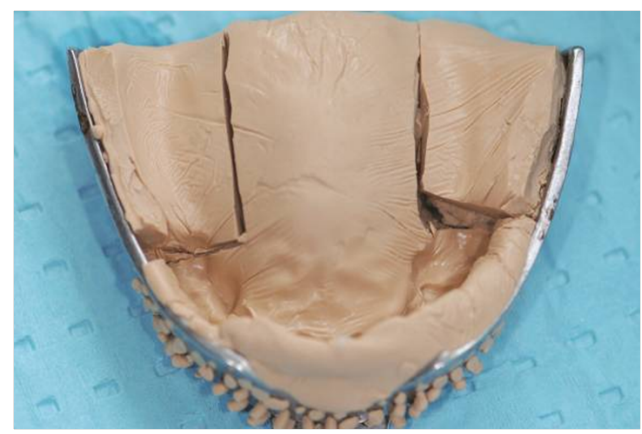

Figure 5. Opposite-tooth biting on the bar of the force transport during the final impression making (Case 1).

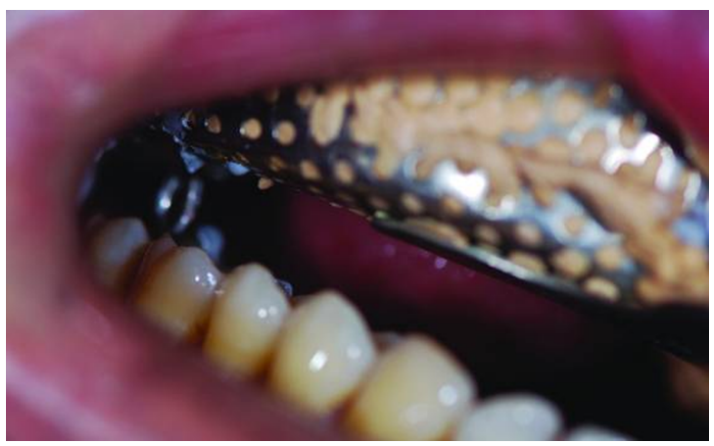

Figure 6. Final impression (Case 1).

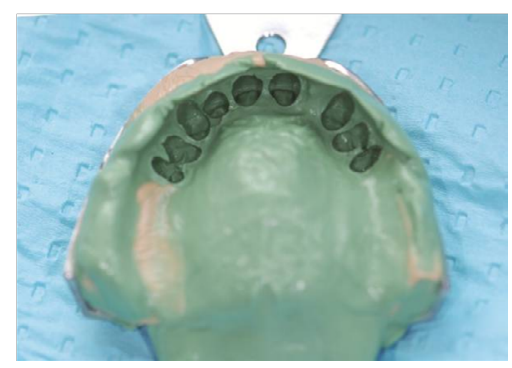

Figure 7. Fixed part of the attachment denture tried on (Case 2).
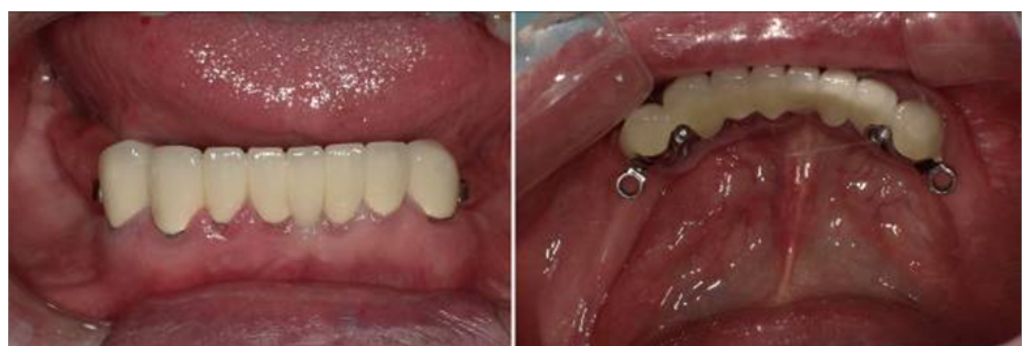
Figure 8. Force transport unit assembled on the perforated stock tray (Case 1); (a) tissue surface view; (b) outside view (Case 2).

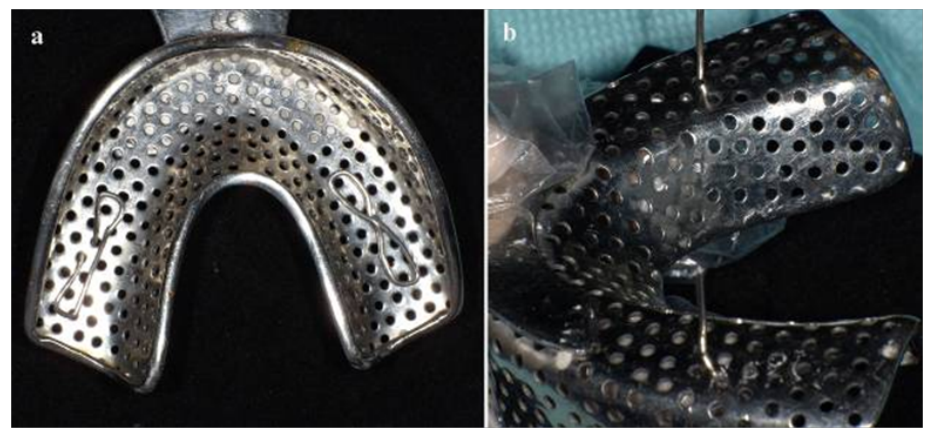

Figure 9. Preliminary impression made with a heavy body-type of vinyl polysiloxane impression materials; (a) tissue surface view; (b) outside view (Case 2).
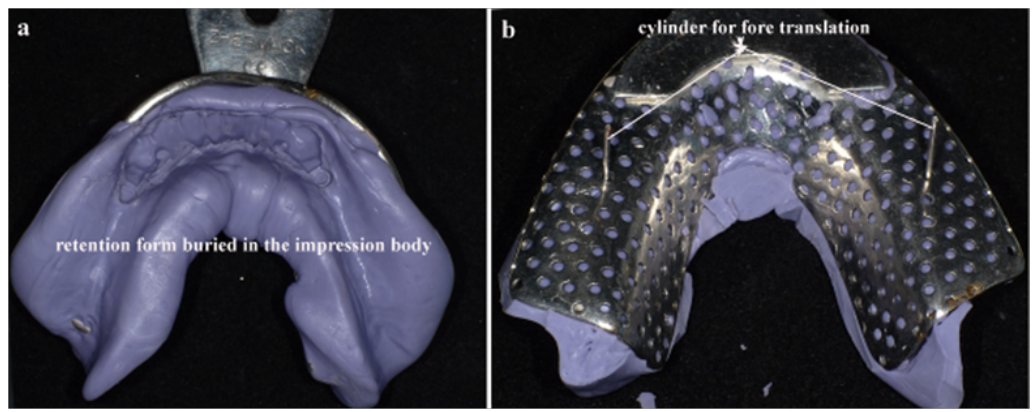

Figure 10. Part of the impression overlapping the residual ridge free from the whole impression body and the tray with a scalpel (Case 2); (a) saddle areas separated with a scalpel; (b) saddle area separated from the impression body and the tray.

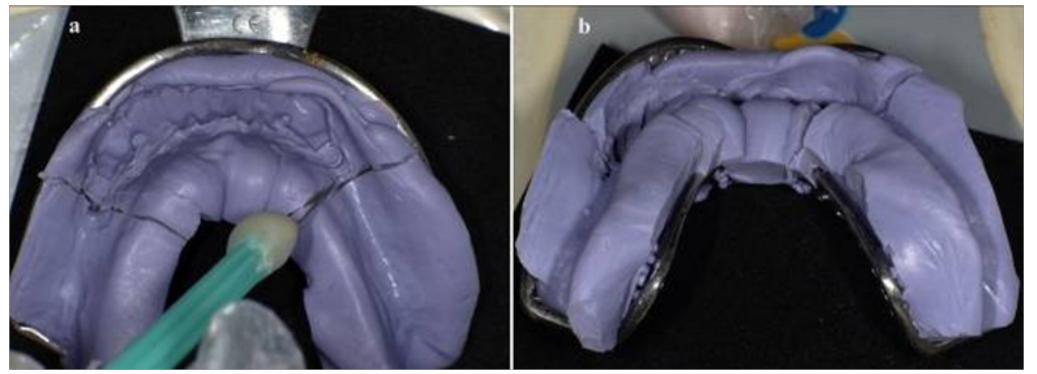

Figure 11. Opposite-tooth biting on the bar of the force transport during final impression making (Case 2); (a) right; (b) left.
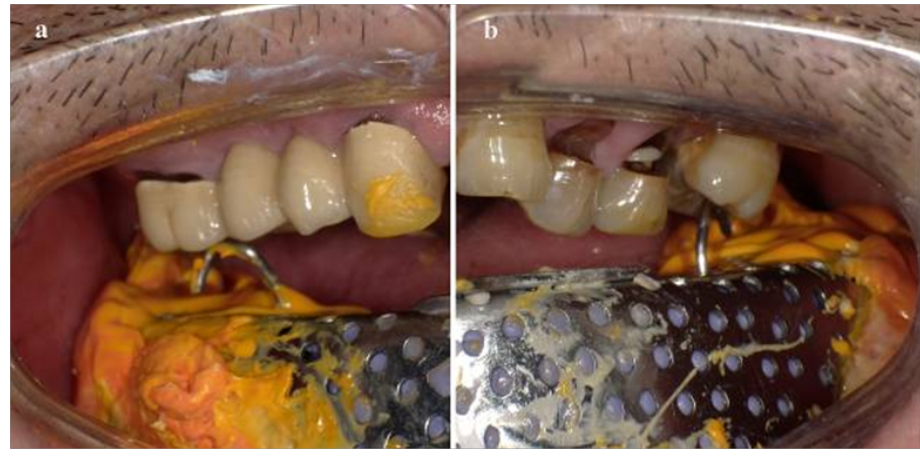

Figure 12. Final impression (Case 2); (a) tissue surface view; (b) outside view (Case 2).
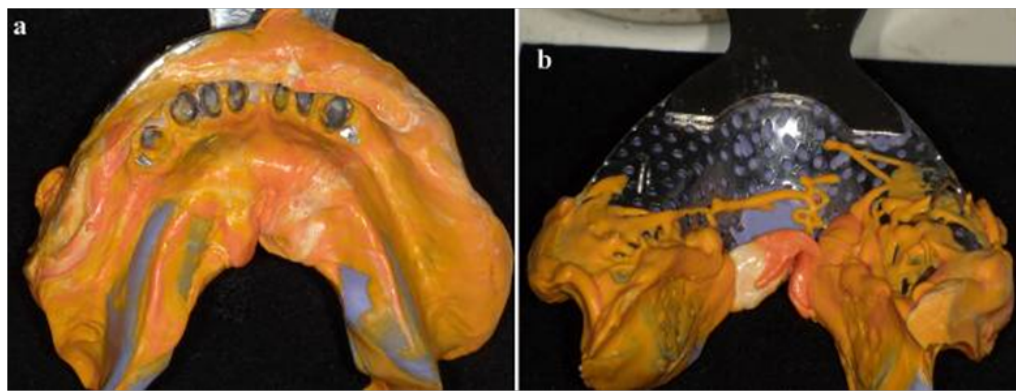


\section{Discussion}

Compared with the altered master cast technique, the method presented in this study is very simple, accurate, and time-efficient for dentists and patients. Moreover, the proposed method does not require supplemental laboratory work. In the altered master cast technique, the metal framework is fabricated with an anatomic impression other than the functional impression. Thus, if the circumferential clasp is used on the abutment, the amount of extra-pivoting force on abutments cannot be avoided, which is the same problem encountered with the traditional anatomic impression technique. However, the metal framework of the proposed method is fabricated with full arch impression, which relates the anatomic form of the teeth to the functional form of the residual ridges in distal extension. Thus, the extra-pivoting force on abutments is decreased. Moreover, our procedure uses a lightbody PVS impression material for the final impression with low viscosity property. Compared with the altered master cast technique, which uses a self-curing acrylic resin with high viscosity, the proposed technique may accurately reproduce the morphology of the residual ridge. The effect of the viscosity of the impression materials has been proven in vitro [13].

The following characteristics should be emphasised in the proposed method: (1) This method must evenly transport the masticatory force to the residual ridge to design the application for masticatory force transport. Thus, the retention form that is buried into the primary impression should span over the ridge from medial to distal positions, and its force-transmitting bar should be located at the central ridge from medial to distal positions, as well as from buccal to lingual positions. The retention form should be made large enough to disperse the masticatory force on the ridge. (2) The portion of the impression that overlaps the ridge is separated from the whole body of the primary impression. The ridge is mentally outlined and thoroughly cut along its boundary. "The section impression overlapping the residual ridge" must be displaced freely under the vertical masticatory force and retained in situ and with relative stability with the tray. (3) Elastomeric impression material should be selected, such as polyvinyl siloxane impression material or polyether material. Hydrocolloid impression material should be avoided in this method.

The proposed method should be classified as a high pressure, mucodisplasive impression. Specifically, pressure under a close fitting surface in the technique is greater than that under a spaced impression tray, which has been proven in vitro [13]. However, the mucodisplasive high pressure impression techniques have been thought to restrict blood supply to the bone, thereby increasing alveolar resorption [13]. Clinical trials have shown that the altered master cast technique does not offer significant advantages [14]. Thus, to improve the credibility of this current technique, the proposed technique must be continuously investigated in vitro and in a cross-over randomised controlled clinical trial, for which suitable protocols are published [15].

\section{Summary}

A novel functional pressure impression procedure for distal-extension RPD is presented. This method can record the anatomic form of the teeth and functional form of the residual ridges simultaneously and accurately. This procedure is simple, can be easily mastered by dentists, and does not increase laboratory workload of dental technicians. However, its clinical effect needs to be proven with a randomised controlled clinical trial.

\section{References}

[1]. Monteith BD (1984) Management of loading forces on mandibular distal extension prostheses. Part 1: evaluation of concepts for design. J Prosthet Dent 52(5): 673-681.

[2]. Kyuho Lee (1996) Double impression procedure for removable partial denture retained with semiprecision attachment: A clinical report. J Prosthet Dent 75(6): 583-587.

[3]. Giffin KM (1996) Solving the distal extension removable partial denture base movement dilemma: A clinical report. J Prosthet Dent 76(4): 347-349.

[4]. El-Sheikh HA, Abdel-Hakim AM (1998) Sectional impressions for mandibular distal extension removable partial dentures. J Prosthet Dent 80(2): 216-219.

[5]. Holmes JB (2001) Influence of impression procedures and occlusal loading on partial denture movement. J Prosthet Dent 86(4): 335-341.

[6]. Applegate OC (1959) Essentials of Removable Partial Denture Prosthesis. (2nd edn), W. B. Saunders Company, Philadelphia. 230-244.

[7]. Hindels GW (1952) Load Distribution in Extension Saddle Partial Dentures. J Prosthet Dent 85(4): 324-329.

[8]. Leupold RJ, Kratochvil FJ (1965) An altered-cast procedure to improve tissue support for removable partial dentures. J Prosthet Dent 15(4): 672-678.

[9]. Lay L-S, Lai W-H, Wu CT (1996) Making the framework try-in, alteredcast impression, and occlusal registration in one appointment. J Prosthet Dent 75(4): 446-448.

[10]. Santana-Penín U, Lozano JG (1998) An accurate method for occlusal registration and altered-cast impression for removable partial dentures during the same visit as the framework try-in. J Prosthet Dent 80(5): 615-618.

[11]. Steven K Nelson, Gregory R Parr (1997) Maintaining proper framework/ altered master cast relationship when processing the distal extension removable partial denture: A simple techbique. J Prosthet Dent 77(6): 639- 640.

[12]. Herman B Dumbrigue, Josephine F Esquivel (1998) Selective-pressure single impression procedure for tooth-mucosa-supported removable partial dentures. J Prosthet Dent 80(2): 259-261.

[13]. Hyde, Timothy Paul (2011) A cross-over randomised controlled trial of selective pressure impressions for lower complete dentures and laboratory investigations into impression pressure variation. $\mathrm{PhD}$ thesis, University of Leeds. http://etheses.whiterose.ac.uk/1761.

[14]. Frank RP, Brudvik JS, Noonan CJ (2004) Clinical outcome of the altered cast impression procedure compared with use of a one-piece cast. J Prosthet Dent 91(5): 468-476.

[15]. Gray JC, Navarro-Coy N, Pavitt SH, Hulme C, Godfrey M, et al. (2012) Improvdent: Improving dentures for patient benefit. A crossover randomised clinical trial comparing impression materials for complete dentures. BMC Oral Health 12(1): 37 . 